\title{
The Aminocarbonylation of Aryl Halides Using Nickel Phosphite Catalytic System
}

\author{
Jinhun Ju $^{\dagger}$, Miso Jeong ${ }^{\dagger}$, Jeongju Moon ${ }^{\dagger}$, Hyun Min Jung ${ }^{\ddagger}$, Sunwoo Lee ${ }^{\dagger *}$ \\ ${ }^{\dagger}$ Department of Chemistry, Chonnam National University, \\ and ${ }^{*}$ Advanced Materials Division, Korea Research Institute of Chemical Technology, \\ Republic of Korea
}

\section{Supporting Information}

\section{Table of Contents}

Page

General Procedure

General Procedure for Nickel Catalyzed Aminocarbonylation 


\section{General Procedure}

The reagents and solvents were obtained from commercial sources and were generally used without further purification. Naphthalene was used as a standard in the quantitative GC experiments using response factors determined from isolated products. All NMR spectra were recorded in $\mathrm{CDCl}_{3}$ with a Bruker AM 300 spectrometer. Chemical shifts are given in $\delta$ ppm downfield from $\mathrm{Me}_{4} \mathrm{Si}\left(\delta 0,{ }^{1} \mathrm{H}\right)$ or $\mathrm{CDCl}_{3}\left(\delta 77,{ }^{13} \mathrm{C}\right)$ as an internal standard. Elemental analyses were performed by the Center for Biofunctional Molecules, Pohang University of Science and Technology.

General Procedure for Nickel Catalyzed Aminocarbonylation: $\mathrm{Ni}(\mathrm{OAc})_{2} \cdot 4 \mathrm{H}_{2} \mathrm{O}(24.9 \mathrm{mg}, 0.1 \mathrm{mmol})$, phosphite 1 (64.7 mg, $0.1 \mathrm{mmol})$ and aryl halide $(2.0 \mathrm{mmol})$ were combined with $\mathrm{NaOMe}(432 \mathrm{mg}, 8.0$ $\mathrm{mmol})$ in a small round-bottom flask. 1,4-Dioxane $(6.0 \mathrm{ml})$ and DMF $(1.0 \mathrm{ml})$ were added, and the flask was sealed with a septum. The resulting mixture was placed in an oil bath at $110{ }^{\circ} \mathrm{C}$ until the starting material was consumed, as determined by GC and TLC. The reaction was poured into $20 \mathrm{~mL}$ of saturated aqueous ammonium chloride and extracted ( $3 \times 20 \mathrm{~mL})$ with $\mathrm{Et}_{2} \mathrm{O}$. The combined ether extracts were washed with brine $(60 \mathrm{~mL})$, dried over $\mathrm{MgSO}_{4}$, and filtered. The solvent was removed under vacuum, and the resulting crude product was purified by flash chromatography on silica gel. The product was eluted with $80 \%$ ethyl acetate in hexane.

\section{4-Methoxy- $\boldsymbol{N}, \boldsymbol{N}$-dimethylbenzamide ${ }^{1}$ (Table 3 , entries 1 and 11 )}

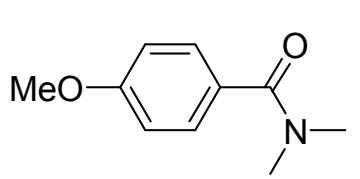

1-Iodo-4-methoxybenzene (468 mg, $2.0 \mathrm{mmol}$, entry 1) or 1-bromo-4methoxybenzene (374 $\mathrm{mg}, 2.0 \mathrm{mmol}$, entry 11) as aryl halide was treated according to general procedure to give 4-methoxy- $N, N$-dimethylbenzamide (354 $\mathrm{mg}, 1.97 \mathrm{mmol}, 99 \%$ yield in entry 1 and $222 \mathrm{mg}, 1.24 \mathrm{mmol}, 62 \%$ yield in entry

11). ${ }^{1} \mathrm{H} \mathrm{NMR}\left(\mathrm{CDCl}_{3}, 300 \mathrm{MHz}\right) \delta 7.40(\mathrm{~d}, J=8.7 \mathrm{~Hz}, 2 \mathrm{H}), 6.90(\mathrm{~d}, J=8.7 \mathrm{~Hz}, 2 \mathrm{H}), 3.82(\mathrm{~s}, 3 \mathrm{H}), 3.05$ (br s, 6H). ${ }^{13} \mathrm{C} \mathrm{NMR}\left(\mathrm{CDCl}_{3}, 75 \mathrm{MHz}\right) \delta 171.43,160.52,129.05,128.38,113.47,55.25,39.68,35.45$.

\section{4, $N, N$-Trimethylbenzamide ${ }^{1}$ (Table 3 , entries 2 and 7)}

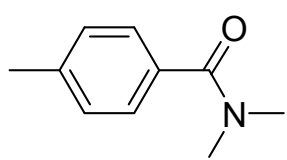

1-Iodo-4-methylbenzene (436 mg, $2.0 \mathrm{mmol}$, entry 2) or 1-bromo-4-methylbenzene (342 $\mathrm{mg}, 2.0 \mathrm{mmol}$, entry 7) as aryl halide was treated according to general procedure to give 4, $\mathrm{N}, \mathrm{N}$-trimethylbenzamide $(320 \mathrm{mg}, 1.96 \mathrm{mmol}, 98 \%$ yield in entry 2 and $324 \mathrm{mg}, 1.99 \mathrm{mmol}, 99 \%$ yield in entry 13$).{ }^{1} \mathrm{H}$ NMR $\left(\mathrm{CDCl}_{3}, 300\right.$ $\mathrm{MHz}) \delta 7.31(\mathrm{~m}, 2 \mathrm{H}), 7.19(\mathrm{~m}, 2 \mathrm{H}), 3.09$ (br s, 3H), 2.99 (br s, 3H), 2.37 (s, 3H). ${ }^{13} \mathrm{C} \mathrm{NMR}\left(\mathrm{CDCl}_{3}, 75\right.$ MHz) $\delta 171.78,139.57,133.35,128.88,127.16,39.64,35.38,21.35$. 
2,N,N-Trimethyl-benzamide ${ }^{1}$ (Table 3 , entry 3 )

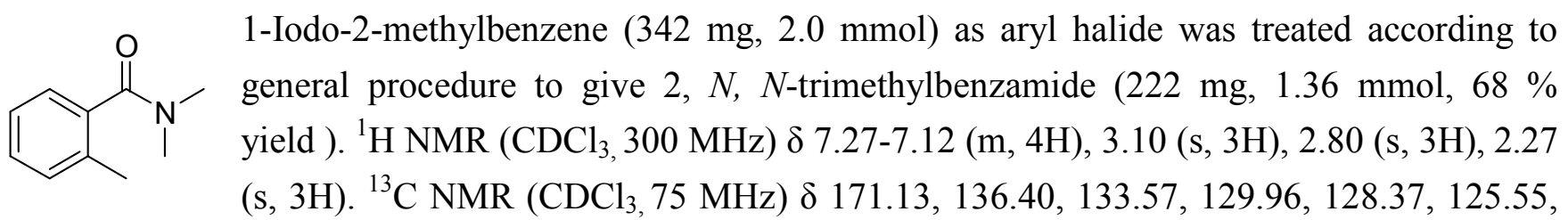
$125.41,37.99,34.14,18.52$.

Naphthalene-1-carboxylic acid dimethylamide ${ }^{1}$ (Table 3, entries 4 and 14)

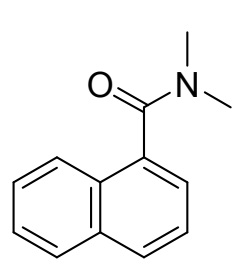

1-Iodonaphthalene (508 mg, $2.0 \mathrm{mmol}$, entry 4) or 1-bromonaphthalene (414 mg, 2.0 mmol, entry 14) as aryl halide was treated according to general procedure to give naphthalene-1-carboxylic acid dimethylamide (179 mg, $0.9 \mathrm{mmol}, 45 \%$ yield in entry 4 and $255 \mathrm{mg}, 1.28 \mathrm{mmol}, 64 \%$ yield in entry 14$).{ }^{1} \mathrm{H}$ NMR $\left(\mathrm{CDCl}_{3}, 300 \mathrm{MHz}\right) \delta 7.86-$ 7.82 (m, 2H), 7.79-7.75 (m, 1H), 7.52-7.44 (m, 3H), 7.43-7.37 (m, 1H), 3.22 (s, 3H), $2.76(\mathrm{~s}, 3 \mathrm{H}) .{ }^{13} \mathrm{C} \mathrm{NMR}\left(\mathrm{CDCl}_{3}, 75 \mathrm{MHz}\right) \delta 170.65,134.58,133.25,129.28,128.79,128.20,126.75$, $126.14,124.99,124.65,123.66,38.63,34.63$.

$\mathbf{N}, \mathbf{N}$-dimethyl-4-trifluoromethyl-benzamide ${ }^{1}$ (Table 3 , entry 5 )<smiles>CN(C)C(=O)c1ccc(C(F)(F)F)cc1</smiles>

1-Iodo-4-trifluoromethxylbenzene $(544 \mathrm{mg}, 2.0 \mathrm{mmol}$ ) as aryl halide was treated according to general procedure to give $N, N$-dimethyl-4-trifluoromethyl-benzamide (48 mg, $0.22 \mathrm{mmol}, 11 \%$ yield, $\sim 90 \%$ purity). ${ }^{1} \mathrm{H}$ NMR $\left(\mathrm{CDCl}_{3}, 300 \mathrm{MHz}\right) \delta 7.67$ (d, $J=7.8 \mathrm{~Hz}, 2 \mathrm{H}), 7.53$ (d, $J=7.8 \mathrm{~Hz}, 2 \mathrm{H}), 3.13(\mathrm{~s}, 3 \mathrm{H}), 2.90(\mathrm{~s}, 3 \mathrm{H}) .{ }^{13} \mathrm{C} \mathrm{NMR}$ $\left(\mathrm{CDCl}_{3}, 75 \mathrm{MHz}\right) \delta 170.06,139.83,131.39\left(\mathrm{q}, J_{C-F}=32.8 \mathrm{~Hz}\right), 127.33,125.45\left(\mathrm{~d}, J_{C-F}=3.8 \mathrm{~Hz}\right)$, $125.35\left(\mathrm{~d}, J_{C-F}=3.8 \mathrm{~Hz}\right), 39.30,35.26$.

\section{$N, N$-Dimethylbenzamide ${ }^{2}$ (Table 3 , entry 6 )}

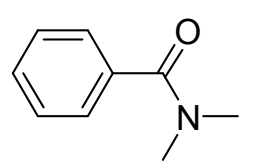

Bromobenzene $(314 \mathrm{mg}, 2.0 \mathrm{mmol})$ as aryl halide was treated according to general procedure to give $N, N$-Dimethylbenzamide (292 mg, $0.96 \mathrm{mmol}, 98 \%$ yield). ${ }^{1} \mathrm{H}$

NMR $\left(\mathrm{CDCl}_{3}, 300 \mathrm{MHz}\right) \delta$ 7.43-7.36 (m, 5H), 3.11 (br s, 3H), 2.97 (br s, $\left.3 \mathrm{H}\right) .{ }^{13} \mathrm{C}$ $\operatorname{NMR}\left(\mathrm{CDCl}_{3}, 75 \mathrm{MHz}\right) \delta 171.55,136.29,129.41,128.26,126.96,39.48,35.23$.

4-tert-Butyl- $\boldsymbol{N}, \boldsymbol{N}$-dimethyl-benzamide (Table 3 , entry 8 )

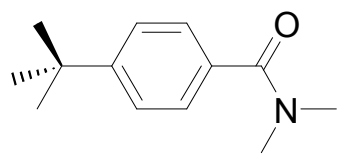

1-Bromo-4-tert-butylbenzene $(426 \mathrm{mg}, 2.0 \mathrm{mmol}$ ) as aryl halide was treated according to general procedure to give 4-tert-butyl- $N, N$-dimethyl-benzamide (382 mg, 1.86 mmol, $93 \%$ yield). Mp $72 \sim 74{ }^{\circ} \mathrm{C} .{ }^{1} \mathrm{H}$ NMR $\left(\mathrm{CDCl}_{3}, 300 \mathrm{MHz}\right) \delta 7.42-$ 
7.33 (m, 4H), 3.09 (br s, 3H), 3.00 (br s, 3H), 1.32 (s, 9H). ${ }^{13} \mathrm{C} \mathrm{NMR}\left(\mathrm{CDCl}_{3}, 75 \mathrm{MHz}\right) \delta 171.75,152.64$, 133.31, 126.89, 125.15, 39.58, 35.32, 34.71, 31.16. IR (NaCl, $\left.\mathrm{cm}^{-1}\right)$ 2961(w), 1634 (s), 1394 (w). Mass (EI): $205(\mathrm{M}+, 21), 161$ (100), 91 (10). Anal Calcd for $\mathrm{C}_{13} \mathrm{H}_{19} \mathrm{NO}$ : C, 76.06; H, 9.33; N, 6.82. found: C, $76.43 ; \mathrm{H}, 9.17 ; \mathrm{N}, 6.62$.

2-Methoxy- $\boldsymbol{N}, \boldsymbol{N}$-dimethylbenzamide ${ }^{3}$ (Table 3, entry 9)

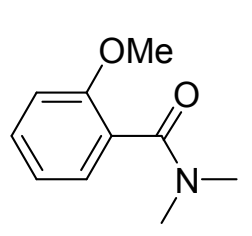

2-Bromo-methoxybenzene (374 $\mathrm{mg}, 2.0 \mathrm{mmol}$ ) as aryl halide was treated according to general procedure to give 2-methoxy- $N, N$-dimethylbenzamide (355 $\mathrm{mg}, 1.98 \mathrm{mmol}$, $99 \%$ yield). ${ }^{1} \mathrm{H}$ NMR $\left(\mathrm{CDCl}_{3}, 300 \mathrm{MHz}\right) \delta 7.33$ (ddd, $\left.J=9.3,8.4,1.8 \mathrm{~Hz}, 1 \mathrm{H}\right), 7.23$ $(\mathrm{dd}, J=7.5,1.8 \mathrm{~Hz}, 1 \mathrm{H}), 6.97(\mathrm{td}, J=7.5,0.9 \mathrm{~Hz}, 1 \mathrm{H}), 6.91(\mathrm{~d}, J=8.4 \mathrm{~Hz}, 1 \mathrm{H}), 3.83(\mathrm{~s}$, $3 \mathrm{H}), 3.11(\mathrm{~s}, 3 \mathrm{H}), 2.84(\mathrm{~s}, 3 \mathrm{H}) .{ }^{13} \mathrm{C} \mathrm{NMR}\left(\mathrm{CDCl}_{3}, 75 \mathrm{MHz}\right) \delta 169.36,155.26,130.20,127.83,126.32$, $120.82,110.88,55.51,38.17,34.68$.

3-Methoxy- $\boldsymbol{N}, \boldsymbol{N}$-dimethylbenzamide ${ }^{3}$ (Table 3 , entry 10 )

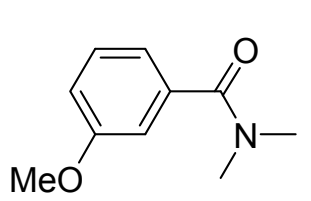

3-Bromo-methoxybenzene $(374 \mathrm{mg}, 2.0 \mathrm{mmol}$ ) as aryl halide was treated according to general procedure to give 3-methoxy- $N, N$-dimethylbenzamide (294 mg, 1.64 mmol, $82 \%$ yield). ${ }^{1} \mathrm{H} \mathrm{NMR}\left(\mathrm{CDCl}_{3}, 300 \mathrm{MHz}\right) \delta$ 7.32-7.26 (m, 1H), 6.97-6.91 (m, $3 \mathrm{H}), 3.81$ (s, 3H), 3.09 (br s, 3H), 2.97 (br s, 3H). ${ }^{13} \mathrm{C} \mathrm{NMR}\left(\mathrm{CDCl}_{3}, 75 \mathrm{MHz}\right) \delta$ $171.20,159.38,137.52,129.29,118.98,115.22,112.21,55.17,39.36,35.12$.

\section{Biphenyl-2-carboxylic acid dimethylamide ${ }^{4}$ (Table 3, entry 12)}

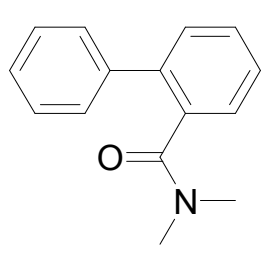

2-Bromobiphenyl (466 mg, $2.0 \mathrm{mmol}$ ) as aryl halide was treated according to general procedure to give biphenyl-2-carboxylic acid dimethylamide (392 $\mathrm{mg}, 1.74 \mathrm{mmol}$, $87 \%$ yield). ${ }^{1} \mathrm{H}$ NMR $\left(\mathrm{CDCl}_{3}, 300 \mathrm{MHz}\right) \delta$ 7.85-7.44 (m, 2H), 7.43-7.37 (m, 5H), 7.37-7.33 (m, 2H), 2.83 (br s, 3H), 2.38 (br s, 3H). ${ }^{13} \mathrm{C} \mathrm{NMR}\left(\mathrm{CDCl}_{3}, 75 \mathrm{MHz}\right) \delta$ 171.22 , 139.83, 138.58, 135.66, 129.21, 128.37, 128.27, 127.59, 127.49, 127.30,

$37.83,34.42$.

Biphenyl-4-carboxylic acid dimethylamide (Table 3, entry 13)

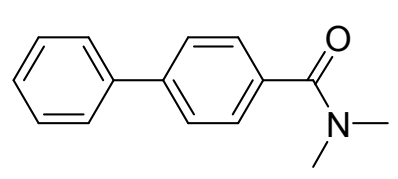

4-Bromobiphenyl (466 mg, $2.0 \mathrm{mmol}$ ) as aryl halide was treated according to general procedure to give biphenyl-4-carboxylic acid dimethylamide (383 $\mathrm{mg}$, $1.70 \mathrm{mmol}, 85 \%$ yield $) . \mathrm{Mp} 103 \sim 105{ }^{\circ} \mathrm{C} .{ }^{1} \mathrm{H} \mathrm{NMR}\left(\mathrm{CDCl}_{3}, 300 \mathrm{MHz}\right) \delta 7.64-$ $7.57(\mathrm{~m}, 4 \mathrm{H}), 7.51-7.42(\mathrm{~m}, 4 \mathrm{H}), 7.39-7.33(\mathrm{~m}, 1 \mathrm{H}), 3.13(\mathrm{br} \mathrm{s}, 3 \mathrm{H}), 3.04$ (br s, 3H). ${ }^{13} \mathrm{C} \mathrm{NMR}\left(\mathrm{CDCl}_{3}, 75 \mathrm{MHz}\right) \delta 171.43,142.39,140.31,135.05,128.84,127.69,127.63,127.11$, 
127.02, 39.64, 35.37. IR ( $\left.\mathrm{NaCl}, \mathrm{cm}^{-1}\right) 3011$ (w), 2930 (w), 1616 (s), 1398 (m). MS (EI): 225 (M+, 32), 181 (100), 152 (61). Anal Calcd for $\mathrm{C}_{15} \mathrm{H}_{15} \mathrm{NO}$ : C, 79.97; H, 6.72; N, 6.22. found: $\mathrm{C}, 79.48 ; \mathrm{H}, 6.74 ; \mathrm{N}$, 6.17 .

\section{Naphtalene-2-carboxylic acid dimethylamide ${ }^{5}$ (Table 3, entry 15)}<smiles>CN(C)C(=O)c1ccc2ccccc2c1</smiles>

2-Bromonaphthalene (414 $\mathrm{mg}, 2.0 \mathrm{mmol}$ ) as aryl halide was treated according to general procedure to give naphtalene-2-carboxylic acid dimethylamide $(287 \mathrm{mg}$, $1.44 \mathrm{mmol}, 72 \%$ yield). ${ }^{1} \mathrm{H} \mathrm{NMR}\left(\mathrm{CDCl}_{3}, 300 \mathrm{MHz}\right) \delta 7.89(\mathrm{~s}, 1 \mathrm{H}), 7.85-7.81(\mathrm{~m}$, $3 \mathrm{H}), 7.51-7.48(\mathrm{~m}, 3 \mathrm{H}), 3.13$ (br s, 3H), 2.99 (br s, 3H). ${ }^{13} \mathrm{C}$ NMR $\left(\mathrm{CDCl}_{3}, 75\right.$

MHz) $\delta 171.41,133.47,133.40,132.47,128.19,127.98,127.59,126.78,126.64,126.43,124.24,39.45$, 35.23 .

6-Methoxy-naphtalene-2-carboxylic acid dimethylamide ${ }^{6}$ (Table 3, entry 16)<smiles>COc1ccc2cc(C(=O)N(C)C)ccc2c1</smiles>

2-Bromo-6-methoxynaphthalene $(474 \mathrm{mg}, 2.0 \mathrm{mmol}$ ) as aryl halide was treated according to general procedure to give 6-Methoxy-naphtalene-2carboxylic acid dimethylamide (445 mg, $1.94 \mathrm{mmol}, 97 \%$ yield). ${ }^{1} \mathrm{H}$ NMR $\left(\mathrm{CDCl}_{3}, 300 \mathrm{MHz}\right) \delta 7.83(\mathrm{~d}, J=1.2 \mathrm{~Hz}, 1 \mathrm{H}), 7.73(\mathrm{~d}, J=8.7 \mathrm{~Hz}, 2 \mathrm{H})$, 7.47 (dd, $J=8.4,1.5 \mathrm{~Hz}, 1 \mathrm{H}), 7.18-7.11(\mathrm{~m}, 2 \mathrm{H}), 3.89$ (s, 3H), 3.10 (br s, $3 \mathrm{H}), 3.03$ (br s, $3 \mathrm{H}) .{ }^{13} \mathrm{C}$ NMR $\left(\mathrm{CDCl}_{3}, 75 \mathrm{MHz}\right) \delta 171.58,158.32,134.83,131.12,129.70,127.84,126.68,126.65,124.93$, $119.32,105.48,55.13,39.49,35.31$.

Thiophene-2-carboxylic acid dimethylamide ${ }^{7}$ (Table 3, entry 17)

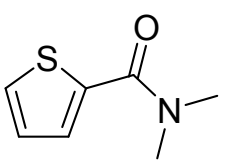

$\mathrm{Ni}(\mathrm{OAc})_{2} \cdot 4 \mathrm{H}_{2} \mathrm{O}(49.8 \mathrm{mg}, 0.2 \mathrm{mmol})$, phosphite 1 (129.4 mg, $\left.0.2 \mathrm{mmol}\right)$ and 2bromothiophene (326 mg, $2.0 \mathrm{mmol}$ ) as aryl halide were treated according to general procedure to give 2-methoxy- $N, N$-dimethylbenzamide (292 $\mathrm{mg}, 1.88 \mathrm{mmol}, 94 \%$ yield). ${ }^{1} \mathrm{H}$ NMR $\left(\mathrm{CDCl}_{3}, 300 \mathrm{MHz}\right) \delta 7.44(\mathrm{dd}, J=5.1,0.9 \mathrm{~Hz}, 1 \mathrm{H}), 7.35(\mathrm{dd}, J=3.6,0.9 \mathrm{~Hz}$, 1H), 7.03 (dd, $J=5.1,3.6 \mathrm{~Hz}, 1 \mathrm{H}), 3.18$ (br s, $6 \mathrm{H}) .{ }^{13} \mathrm{C} \mathrm{NMR}\left(\mathrm{CDCl}_{3}, 75 \mathrm{MHz}\right) \delta 164.44,137.91,129.13$, $128.75,126.63 .31 .59,29.59$. 


\section{Synthesis of Piperidin-1-yl-p-toly-methanone ${ }^{8}$}

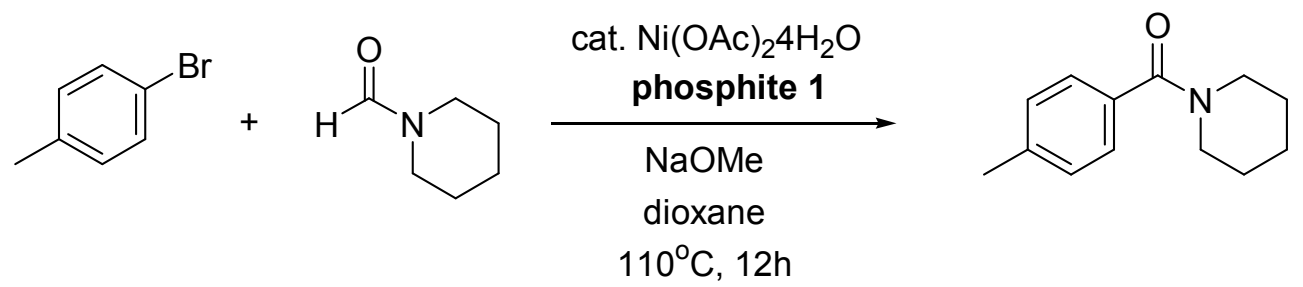

$\mathrm{Ni}(\mathrm{OAc})_{2} \cdot 4 \mathrm{H}_{2} \mathrm{O}(12.4 \mathrm{mg}, 0.05 \mathrm{mmol})$, phosphite 1 (32.3 $\left.\mathrm{mg}, 0.05 \mathrm{mmol}\right)$ and 4-bromomethylbenzne $(171 \mathrm{mg}, 1.0 \mathrm{mmol})$ were combined with $\mathrm{NaOMe}(216 \mathrm{mg}, 4.0 \mathrm{mmol})$ in a small round-bottom flask. 1,4-Dioxane $(4.0 \mathrm{ml})$ and 1-formylpiperidine $(1.131 \mathrm{~g}, 10 \mathrm{mmol})$ were added, and the flask was sealed with a septum. The resulting mixture was placed in an oil bath at $110^{\circ} \mathrm{C}$ for 12 hours. The reaction mixture was poured into $20 \mathrm{~mL}$ of saturated aqueous ammonium chloride and extracted ( $3 \times 20 \mathrm{~mL}$ ) with $\mathrm{Et}_{2} \mathrm{O}$. The combined ether extracts were washed with brine $(60 \mathrm{~mL})$, dried over $\mathrm{MgSO}_{4}$, and filtered. The solvent was removed under vacuum, and the resulting crude product was purified by flash chromatography on silica gel. The product was eluted with 50\% ethyl acetate in hexane. After evaporation of solvent, piperidin-1-yl-p-toly-methanone was obtained with $40.6 \mathrm{mg}(0.2 \mathrm{mmol}, 20 \%$ yield). Mp 53-54 ${ }^{\circ} \mathrm{C}$ (lit 53.5 54 $\left.{ }^{\circ} \mathrm{C}\right) .{ }^{1} \mathrm{H} \mathrm{NMR}\left(\mathrm{CDCl}_{3}, 300 \mathrm{MHz}\right) \delta 7.29$ (d, J=8.1 Hz, 2H), 7.19 (d, $J=$ $8.4 \mathrm{~Hz}, 2 \mathrm{H}), 3.70$ (br, 2H), 3.35 (br, $2 \mathrm{H}), 2.36$ (s, 3H), 1.70-1.40 (br, 6H). ${ }^{13} \mathrm{C} \mathrm{NMR}\left(\mathrm{CDCl}_{3}, 75 \mathrm{MHz}\right) \delta$ $170.48,139.37,133.53,128.94,126.89,48.84,43.16,26.51,25.61,24.61,21.32$.

\section{References}

1) Hosoi, K.; Nozaki, K.; Hiyama, T. Org. Lett. 2002, 4, 2849-2851.

2) Motoyama, Y.; Mitsui, K.; Ishida, T.; Nagashima, H. J. Am. Chem. Soc. 2005, 127, 13150-13151.

3) Yamagiwa, Y.; Ohashi, K.; Sakamoto, Y.; Hirakawa, S.; Kamikawa, T.; Kubo, I. Tetrahedron 1987, 43, 3387-3394.

4) House, H. O.; Bryant, W. M., III. J. Org. Chem. 1966, 31, 3482-3489.

5) Ebden, M. R.; Simpkins, N. S.; Fox, D. N. A. Tetrahedron 1998, 54, 12923-12952.

6) Bindal, R. D.; Katzenellenbogen, J. A. J. Org. Chem. 1987, 52, 3181-3185.

7) Screttas, C. G.; Steele, B. R. J. Org. Chem. 1988, 53, 5151-5153.

8) Otani, Y.; Nagae, O.; Naruse, Y.; Inagaki, S.; Ohno, M.; Yamaguchi, K.; Yamamoto, G.; Uchiyam, M.; Ohwada, T. J. Am. Chem. Soc. 2003, 125, 15191-150199. 